\title{
Photoelastic Apparatus at the California Institute of Technology
}

Cite as: Review of Scientific Instruments 5, 80 (1934); https://doi.org/10.1063/1.1751781 Submitted: 07 November 1933 . Published Online: 27 December 2004

J. H. A. Brahtz

\section{ARTICLES YOU MAY BE INTERESTED IN}

A Method of Producing Uniform Magnetic Fields

Review of Scientific Instruments 5, 78 (1934); https://doi.org/10.1063/1.1751780

\section{Lock-in Amplifiers up to $600 \mathrm{MHz}$}
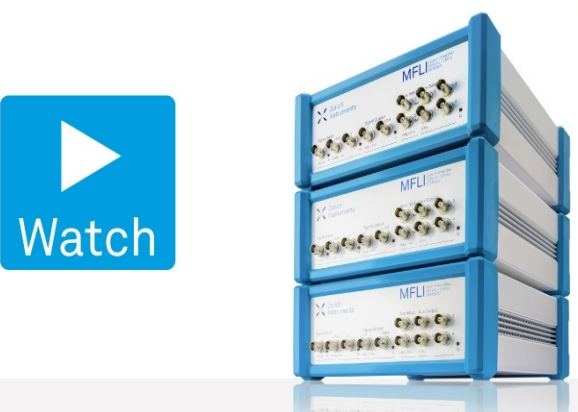


\title{
Photoelastic Apparatus at the California Institute of Technology
}

\author{
J. H. A. BRahtz, California Institute of Technology
}

(Received November 7, 1933)

A description is given of the reflector polariscope built at the California Institute of Technology. The preparation of models and the technique is explained. Examples of systems of isochromatics and isoclinic lines obtained in models of the Pine Canyon Dam, California, are reproduced.
$\mathrm{T}$ HE photoelastic equipment of the California Institute of Technology, initiated by Dr. Th. v. Kármán, differs sufficiently from the conventional arrangement to make a short description worth while.

The primary object in establishing this photoelastic laboratory was to determine experimentally the stress distribution in high gravity dams, especially the influence of the base and the re-entrant corners at the heel and toe. The present writer has made theoretical investigations on this subject ${ }^{1}$ and it was of interest to check the computations by photoelastic experimentation. Very little work has been done by this method on civil engineering structures in which the body forces play an important part. The few investigations were related to slender girders and arches where the body forces are of less importance.

Investigations on dams require models of unusually large dimensions and elaborate loading mechanisms; so it became important to construct an apparatus providing ample space in the optical field for large models and loading systems. Dr. A. Goetz, Professor in Physics at the California Institute of Technology, suggested the use of mirrors in place of the lenses generally found in the conventional type of apparatus, as is described in his own article published in this issue.

The mechanical designs and constructions are due to Dr. A. L. Klein, Mr. Ralph Watson and the writer. The construction of the laboratory and subsequent experimental work was carried

1 Brahtz, Physics 4, 56 (1933); Applied Mechanics 1, No. 2, April-June 31-37 (1933). out in the Guggenheim Graduate School of Aeronautics under the direction of Dr. Th. v. Kármán.

\section{Description of Apparatus}

The light source $L$ consists of a ventilated lantern illuminated by a 1000 watt mazda projection bulb. If monochromatic light is to be employed $L$ consists of a mercury arc operated by a $100 \mathrm{hp} .250$ volt motor-generator with remote control from the photoelastic laboratory. $W$ is a cooling filter consisting of a water-filled glass jar. It removes the heat rays before the light is allowed to pass through the nicol prism $N_{1}$ (polarizer) thus eliminating the danger of melting the Canada balsam joint under prolonged exposure. The $20 \mathrm{~cm}$ reflector $R_{1}$ of $100 \mathrm{~cm}$ focal length transmits parallel light through the test specimen $T$ to a second reflector $R_{2}$, a duplicate of $R_{1}$. From $R_{2}$ the rays converge through a second nicol prism $N_{2}$ (analyzer) to the photographic plate or screen $P$. No objective lens is used. The rays are brought to focus on the plate or screen and any photographic scale can be obtained merely by moving the specimen $T$ and the plate $P$ to proper relative positions in the field.

$M_{1}$ and $M_{2}$ are mica plates split to a thickness corresponding to an optical displacement of onequarter wave-length of the light used in the experiment. They are used to produce circularly polarized light, needed when isochromatic lines are desired alone, i.e., without the presence of the isoclinic lines. $B$ is a blue filter used in connection with supersensitive panchromatic film.

The use of reflectors conveniently separates the polarizing unit from the analyzing unit in 


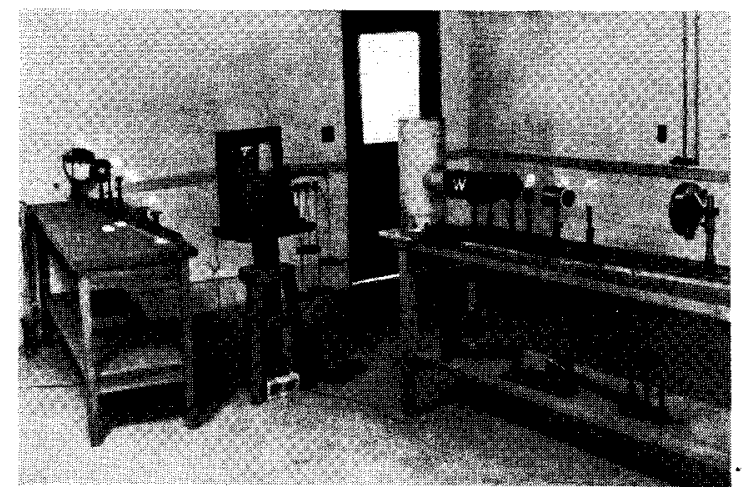

FIG. 1. Photoelastic laboratory at the California Institute of Technology, 1933.

such a way that a large operating space is obtained without excessive length of laboratory. It allows excellent control of the optical path and enables the experimenter to manipulate the loading mechanism and simultaneously observe the image of the fringe pattern. It is possible to build a reflector polariscope with a large diameter parallel beam at a reasonable expense. The comparative cost of a reflector and lens of the same diameter and equal optical properties is about $1: 5$, one of the reasons for using reflectors in the large stellar telescopes. The reflectors were manufactured of crown glass, ground and polished to a $200 \mathrm{~cm}$ radius, the surface being accurate within one-eighth wave-length of mercury light. The reflecting surfaces consist of aluminum in place of the usual silver. The process of preparing aluminum mirrors is being developed by Dr. John Strong in connection with the 200-inch reflector now under construction in the astrophysics shop of the California Institute of Technology. A brief description of the method may be of interest.

Aluminum mirrors are prepared by vacuum distillation of pure aluminum from a tungsten helix directly onto the glass mirror surface. The vacuum necessary for this is obtained by oil diffusion pumps in a large metal bell jar and should be maintained to a pressure lower than $1 / 10$ micron mercury. Important features of the technique are to use large tungsten wire to form the helix, to melt the aluminum onto the tungsten in vacuum before the mirror is placed in the apparatus in order to free the metal of oxide gas, to have the glass scrupulously clean and to have the distance between the mirror and the tungsten helix of the order of the diameter of the mirror so as to insure uniform deposit of aluminum.

The reflecting aluminum surface has the property of high and uniform reflection (90 percent) throughout the visible and far onto ultraviolet. It does not tarnish as silver and may be washed with soap and water to clean off dust, finger marks, etc.

\section{Preparation of Models}

The available transparent, isotropic materials suitable for photoelastic experimentation are Bakelite, Japanese phenolite, celluloid and various types of glass. The nature of the experiments, as described in detail below, called for unusually large models of about one-quarter inch thickness. Due to the existence of sharp reentrant corners, causing high stress concentrations, it was necessary to select a material combining great strength and good elastic properties with a high optical sensitivity. Bakelite 47,001 , manufactured by the Bakelite Corporation, was adopted as best suited for the purpose. At first it was thought necessary to prepare the models from the raw Bakelite plates before annealing the same, in order also to remove the stresses that might be produced in the process of planing and cutting out the models. The raw Bakelite plates were of dimensions $12^{\prime \prime} \times 18^{\prime \prime}$ $\times 7 / 16^{\prime \prime}$. After an unsuccessful attempt to anneal the finished models along the usual lines, i.e., by letting the material come to a temperature of about $80^{\circ} \mathrm{C}$, uniform throughout, and then cooling it slowly over a period of twelve hours in a well-controlled, electrically heated oven, it was decided to anneal first the raw Bakelite plates and then prepare the models from the already annealed plates. It was found impossible to remove the color fringes at the re-entrant corners in the finished models. Furthermore, after the material had been semi-plastic, the models were no longer optically plane near the sharp boundary edges, and the form marks of the raw Bakelite would reappear on the machined surfaces.

A special annealing bath was designed so as to be able to observe the behavior of the plates 
in the photoelastic polariscope during the process of annealing.

The annealing bath consisted of a rectangular transparent jar filled with water heated by electric coils. Uniform temperature throughout the bath was insured by a propeller giving ample convection. In this manner good annealing results were obtained but unless the surfaces of the Bakelite plates were extremely well protected between oiled glass plates against direct contact with the hot water, they would become very brittle and difficult to machine. The process of planing and milling the models produced no stresses if the plates were properly supported and if small cuts were taken with very sharp tools.

Glass models prepared from commercial quarter-inch plate glass were used extensively to obtain the isoclinic lines. As a rule it is easy to obtain even a rather large piece of plate glass free from initial stresses. However, this must be ascertained by rotating the specimen in the field of the plane polariscope (with the quarter wave plates removed) before proceeding to prepare the model. There is never enough initial stress to produce isochromatic lines; only initial isoclinics might be observed, in which case the glass must, of course, be rejected. The advantage of glass for large models is that no planing and polishing are required but experience in glass cutting and grinding is very necessary.

The new water white product BT-61,893, manufactured by the Bakelite Corporation, offers good possibilities for photoelastic work. The extra cost of this material will, no doubt, be more than offset by the cost and time lost in machining Bakelite 47,001.

\section{Technique}

In photoelastic analysis two sets of optical lines are produced, namely, isochromatics and isoclinics. Isochromatics are the interference fringes caused by the optical displacements in the model, due to the property of double refraction produced in isotropic materials when placed under stress. Along the same fringe order the maximum shear is constant and can be evaluated by calibration tests. The isoclinic lines are lines of zero illumination occurring at points

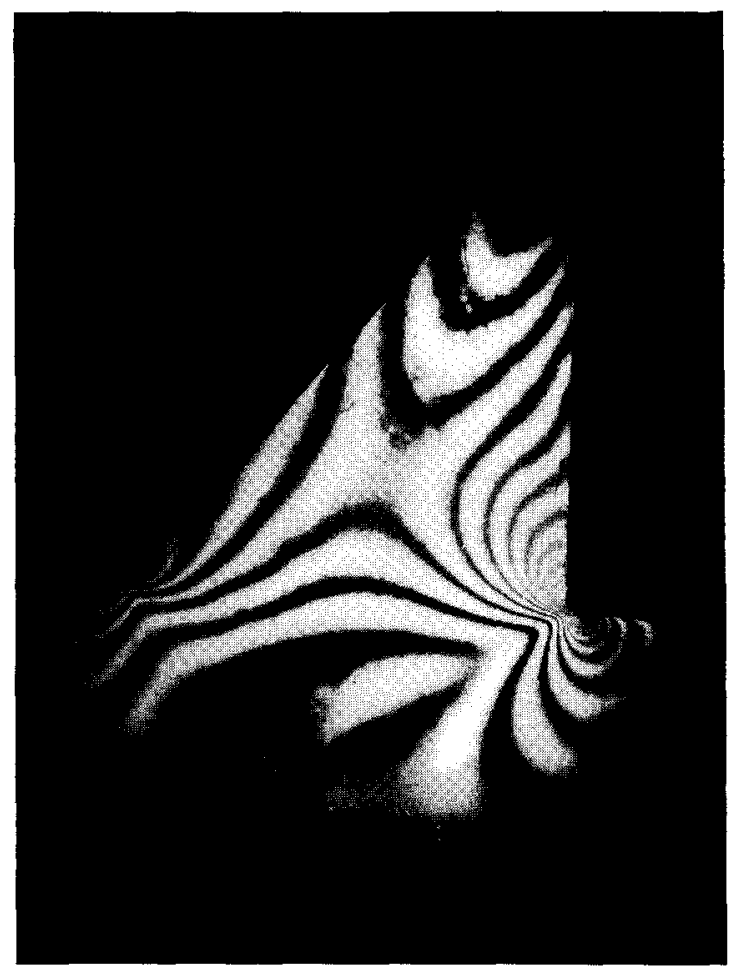

FIG. 2. Isochromatic fringes for hydrostatic loading on model of Pine Canyon Dam, California.

where the direction of principal stress is parallel with the plane of polarization.

Even with complicated stress patterns, it was generally easy to obtain good photographs of the isochromatic lines, both with white and monochromatic light, by insertion of the proper $\frac{1}{4}$ wave plates, i.e., using circularly polarized light. For white light, unless color photographs were taken,

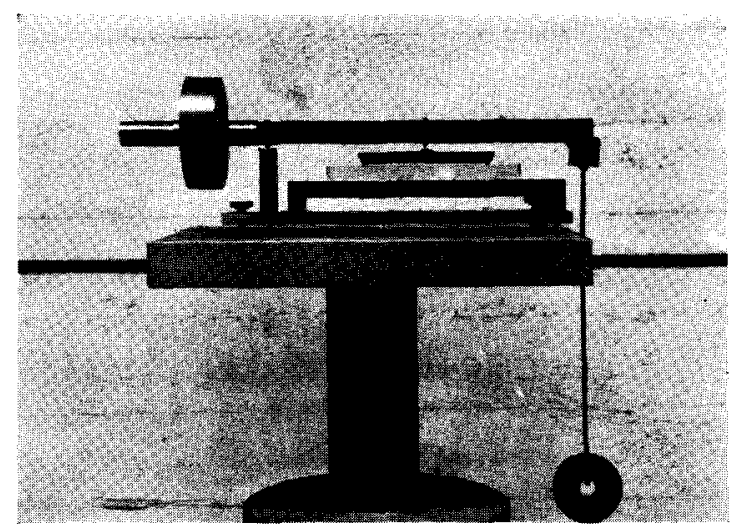

FIG. 3. Bending machine for calibration. 


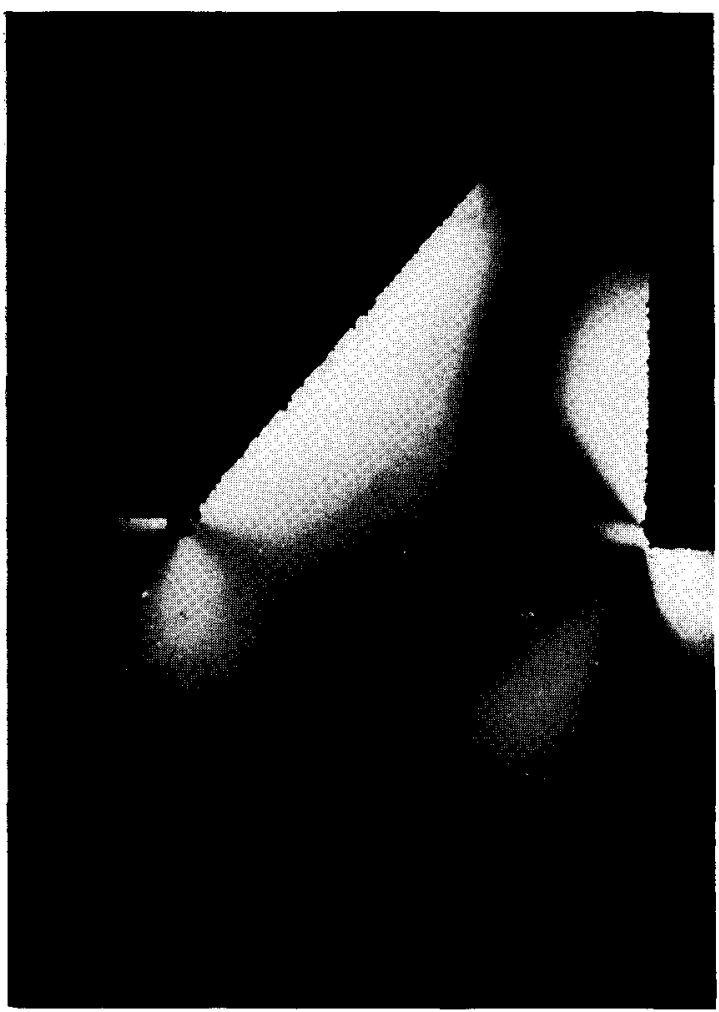

FIG. 4. Isoclinic lines in glass model of Pine Canyon Dam. Same loading as in Fig. 3.

it was convenient to use supersensitive panchromatic film and a blue filter which produces a denser fringe pattern. In the calibration the identical optical set-up was, of course, used. If some initial stresses existed in the model, high enough working stresses could usually be superposed so as to make the final result sufficiently accurate for practical purposes without the need of correcting for initial stresses. ${ }^{2}$ Fig. 2 gives an example of isochromatics (model of the Pine Canyon Dam, hydrostatic loading).

The calibration of the isochromatics was done in the usual manner by subjecting a slender beam to uniform bending. The calibration beam and the test model must be made from the same Bakelite plate. The two should in all respects undergo parallel treatment. The bending machine for calibration is shown in Fig. 3.

It was different with the system of isoclinic lines. In complicated models, especially when several re-entrant corners are present, the isoclinics for each nicol setting will consist of several branches. Unless the model is so free from initial stress that so low a working stress can be used that the isochromatics hardly begin to show up, it becomes a difficult and trying task to separate properly the isoclinic lines from the isochromatics. In such cases glass models proved to be exceedingly helpful. With these a considerable working stress could be used to produce sharp isoclinic lines without the slightest disturbance by isochromatics. An example of isoclinic lines obtained with a glass model of the same object as mentioned above is shown in Fig. 4.

${ }^{2}$ See Photoelasticity by E. G. Coker and L. N. G. Filon, Cambridge, $\$ 3.32,1933$. 